\title{
OMGene: mutual improvement of gene models through optimisation of evolutionary conservation
}

\author{
Michael P. Dunne and Steven Kelly ${ }^{*}$ (D)
}

\begin{abstract}
Background: The accurate determination of the genomic coordinates for a given gene - its gene model - is of vital importance to the utility of its annotation, and the accuracy of bioinformatic analyses derived from it. Currentlyavailable methods of computational gene prediction, while on the whole successful, frequently disagree on the model for a given predicted gene, with some or all of the variant gene models often failing to match the biologically observed structure. Many prediction methods can be bolstered by using experimental data such as RNA-seq. However, these resources are not always available, and rarely give a comprehensive portrait of an organism's transcriptome due to temporal and tissue-specific expression profiles.

Results: Orthology between genes provides evolutionary evidence to guide the construction of gene models. OMGene (Optimise My Gene) aims to improve gene model accuracy in the absence of experimental data by optimising the consistency of multiple sequence alignments of orthologous genes from multiple species. Using RNA-seq data sets from plants, mammals, and fungi, considering intron/exon junction representation and exon coverage, and assessing the intra-orthogroup consistency of subcellular localisation predictions, we demonstrate the utility of OMGene for improving gene models in annotated genomes.
\end{abstract}

Conclusions: We show that significant improvements in the accuracy of gene model annotations can be made, both in established and in de novo annotated genomes, by leveraging information from multiple species.

Keywords: Genome annotation, Annotation errors, Orthogroups, Orthology, Gene model

\section{Background}

The utility of any given genome is dependent on the comprehensiveness and accuracy of its gene model annotations. Inaccuracies in the annotated locations and structures of protein coding genes can lead to myriad downstream errors. These include misinformed conclusions about the biological properties of an organism, as well as errors in transcript quantification, phylogenetic tree inference, protein localisation, and protein structure predictions. It is therefore vital to downstream analysis, both computational and experimental, to ensure that gene annotations are as accurate as possible.

The absolute quantity of publicly available genomic data has grown exponentially over the past two decades, as has

\footnotetext{
* Correspondence: steven.kelly@plants.ox.ac.uk

Department of Plant Sciences, University of Oxford, South Parks Road, Oxford OX1 3RB, UK
}

the number of taxa represented [1-3], owing to the consistently decreasing costs of acquiring whole genome sequences $[4,5]$. Accordingly, the feasibility of manual gene model annotation has diminished progressively, with a corresponding increase in reliance on computational gene prediction software. As such there are numerous tools available for the de novo and data-assisted prediction of genes [6]. These tools typically rely on genetic signatures such as GC content, codon bias, feature length distributions, and various conserved DNA sequence motifs. Though many of these tools are highly proficient at gene prediction, mistakes are common. Gene prediction tools often disagree on the quantity of genes that they predict [7-9]. Furthermore, even when gene predictors agree on the location of a gene, the predicted intron-exon structure for that gene can vary considerably between the different methods [10]. Common errors include erroneous exon/intron retention/omission, inaccurate exon/intron 
boundaries, frame errors, misplaced start codons, and fragmentation/fusion of gene models.

When available, the use of extrinsic empirical data, most notably RNA-seq, is the most reliable currently available method for procuring gene models. For example, single contiguous RNA-seq reads obtained from mRNA sequencing can be split across multiple loci when mapped to the genome, providing evidence for the locations of splice junctions. Unfortunately, empirical data is generally not available for all genes in a given species: many genes are expressed in a cell-type or cell-cycle specific manner and for organisms with many disparate tissue types it can be difficult to obtain RNA-seq data that cover the full breadth of the transcriptome $[11,12]$. In addition, not all gene sequences are amenable to reliable and accurate alignment, in particular identical duplicate genes and genes that contain repetitive regions found in multiple other genes [13]. Furthermore library preparation protocols and other statistical factors can make reliable gene model inferences difficult [14-16]. Finally, there are some aspects of gene models that are simply not revealed by RNA-seq analysis: for example the presence of 5'UTR sequences or internal methionine residues mean that there can often be multiple plausible start codons locations for a given open reading frame (ORF), and so start codon location cannot be inferred from RNA-seq data.

Feature locations (splice sites, exons, transcription start sites) have been shown to be highly conserved across evolutionary timescales, often more so than the constituent amino acid sequences they encapsulate $[17,18]$, despite alternative splicing being a driver of divergence [19]. Given various gene model predictions, if multiple highly similar (in sequence and structure) gene models exist for a gene across multiple taxa, they are more likely to be biologically correct than disparate alternatives. Several de novo gene prediction algorithms have utilised this concept to constrain gene searches by predicting genes in multiple genomes simultaneously: notably SLAM [20], SGP-1 [21], TWINSCAN/N-SCAN/CONTRAST $[22,23]$, and the most recent version of Augustus [24]. However, no tool currently exists that can systematically and automatically improve the annotations of already predicted genes by leveraging annotated gene models from other species.

By considering orthogroups of related genes, one can optimise the similarity of gene models across species by seeking conserved structure across the various taxa. In the absence of extrinsic data, it is parsimonious to choose gene models that maximise intra-orthogroup amino acid alignment agreement. OMGene (Optimise My Gene) aims to improve genome annotations by optimising the agreement between gene models for orthologous genes in multiple species. It is designed to function without the need for additional empirical data, utilising only the local genome sequences for the genes in question, and works on existing predicted gene models. A standalone implementation of the algorithm is available under the GPLv3 licence at https://github.com/ mpdunne/omgene. The algorithm is available as a python script, instructions for which, along with example data sets, are included in the git repository.

\section{Implementation \\ Algorithm description}

The input for OMGene is a set of gene model files in general transfer format (GTF) and a set of corresponding FASTA genome files. GTF is a variant of GFF3 with a more standardised structure. As many public gene datasets use GFF3 format, a tool for extracting wellformed gene entries from GFF3 files is included in the GitHub repository. Each GTF file should contain coordinates for one gene only. The FASTA file can contain one or multiple sequences but must contain the nucleotide sequence referenced by the GTF file. The set of GTF files and their corresponding genome files are inputted to OMGene using a tab-delimited CSV file: each line in the input should contain first the file path to a gene model's GTF file, followed by the path to the genome for that species. If the GTF contains multiple transcript variants then these are considered together as variants of a single gene. Further information regarding input formats can be found in the OMGene GitHub repository.

For each inputted gene, the algorithm defines its gene region to be the region spanning the first and last base of any of its corresponding gene models, with a userselected number of buffer bases either side (default value is $600 \mathrm{bp}$ ). The initial step of OMGene is to cross-align the amino acid sequences from each gene with the gene regions of the other genes using Exonerate [25]. The rationale behind this step is to find exonic regions that are present in one or more gene models but absent from one or more annotated gene model. This is performed three times: first by cross-aligning the input protein sequences against all gene regions, second by crossaligning the protein sequences that have been found in the first step against all gene regions, and finally by cross-aligning all individual exon sequences from the first step. This three-step process mitigates against lack of detection due to gene model errors in one or more of the input genes. This, together with the exons from the original gene sequences, comprises a set of potential gene parts, which may overlap and which may be incompatible in reading frame. Compatible combinations of gene parts (i.e. without frame-shift errors) are strung together to form a putative gene model. Many such putative gene models may exist: the set of putative gene models with the highest alignment score (see Alignment 
score calculation below) is carried forward to the next step. If multiple alternatives have the same alignment score, the choice is made randomly.

The set of putative gene models from the previous step are aligned, and the set of putative exons from all genes is divided into adjacency groups: sets of exons that overlap each other in the alignment (see below). Exons are added in sequentially in these adjacency groups, and at each stage a valid gene model is sought on the left hand side of the gene (i.e. starting at the start codon and seeking to adjoin exons in valid donor-acceptor pairs). Multiple options for each gene are produced at each new junction, by recursively seeking out, or "wiggling" splice junctions (or start codons) in each frame either side of the existing exons start and end points. This produces a set of junction options for each pair of exon ends. A multipartite choice function is then used to choose the optimal set of exons across all secies, as described below. In the event that a particular exon is very small $(<40 \mathrm{bp})$, or does not yield any valid junction sites, both that exon and the one before it are probed for removal, and the variant with the removed exon is compared against the other partial gene models in the evaluation step. Once this recursive step ceases to produce new gene modes, the gene model set with the highest alignment score is declared the winner, and the next putative exon from the next adjacency group is added. If multiple alternatives have the same alignment score, the choice is made randomly. This is repeated until there are no further exons to add.

To ensure that the optimisation process did not overlook potentially better variants in the user-supplied gene models, the process above is repeated. This time, instead of varying exons start and end sites, the set of newly created junctions are compared against the original junctions, aiming to find the optimal combination of new and old junctions.

The final step involves filtering the changes based on a selection of categories that have been observed to over-fix gene models. Firstly, we require the alignment score $\alpha$ of a 10 amino acid region each side of the change to have either remained the same or improved. This is a basic requirement which should be met in most cases due to the way in which sequence variants are chosen. Secondly, changes that have opened gaps in the alignment in three or more of the sequences are not allowed: this is a common occurrence due to sequences proximal to exon termini that by chance feature valid splice junction sequences that are in frame with the adjacent exons and are evolutionarily conserved. These tend not to be correct. Thirdly, very small changes are forbidden: changes that have resulted in two or fewer amino acids being changed in a gapless region of the alignment, such that the new alignment is also gapless, are ignored. Similar changes to larger regions require an $\alpha$ (see Alignment score below) increase of 4 or more. This is to avoid changes that reflect multiple choices of donor-acceptor pairs for essentially identical sequences. Finally, the alignment in the region of the change must be of reasonable quality: for unchanged 5 amino acid regions either side of the region under consideration, the adjusted alignment score $\bar{\alpha}$ must be 3 or higher (or all gaps) for some subset of three sequences containing the sequence of interest. Similarly the resulting score for the changed region must also be higher than 3 or all gaps. Exon boundaries that do not pass the filters are discarded and the genes are reconstructed a final time, allowing only the surviving boundaries and those that were present in the original gene. The resultant genes are outputted in GTF, amino acid FASTA and CDS FASTA format.

\section{Data sources}

For algorithm development and evaluation, three species sets were selected (Table 1): a set of five fungal genomes, a set of five plant genomes, and a set of five mammal genomes. Each species set contained at least one wellannotated model organism. Orthogroups were inferred using OrthoFinder [26]. For the plant data set, where multiple transcript variants were available, the primary transcript was used as listed in Phytozome [27]. For the mammal data set, no primary transcripts were marked, and so for each gene the longest available transcript was used. RNA-seq data sources are listed in Table 2. and were downloaded from the Sequence Read Archive [28].

\section{De novo gene prediction}

De novo gene predictions were made using Augustus [24] version 3.2.2. Training was performed using all well-formed gene models from each species, and using the autoAugTrain.pl script included with the software. Augustus was run individually on each genome with the default settings.

\section{Alignment score}

An amino acid alignment can be considered as an ordered sequence $A=\left(C_{n}\right)_{n=1}^{n=l}$ of columns $C_{n}=\left(c_{1}^{n}, \ldots, c_{l}^{n}\right)$. The column score $\gamma$ for a column $C_{n}$ is defined as the average pairwise Blosum62 score for amino acids in that column:

$$
\gamma\left(C_{n}\right)=\frac{\sum_{1 \leq i<j \leq l} \operatorname{Blos}\left(c_{i}^{n}, c_{j}^{n}\right)}{l}
$$

The Blosum62 matrix was used as it is the most widely used amino acid substitution matrix. The alignment score $\alpha$ for an alignment $A$ is constructed column-wise as:

$$
\alpha(A)=\sum_{n=1}^{l} \gamma\left(C_{n}\right)
$$

The adjusted alignment score $\bar{\alpha}$ is defined as $\bar{\alpha}=\frac{\alpha}{l}$, where $l$ is the alignment length. 
Table 1 Species sets used for algorithm validation

\begin{tabular}{|c|c|c|c|c|c|}
\hline & Species Name & Source & Version/Strain & Taxonomy ID & References \\
\hline \multirow[t]{5}{*}{ Plant species } & Arabidopsis thaliana & $J G l^{1}$ & TAIR10 & 3702 & {$[39,40]$} \\
\hline & Brassica rapa & $J G l$ & v1.3 & 3711 & [27] \\
\hline & Carica papaya & $J G l$ & ASGPBV0.4 & 3649 & {$[39,41]$} \\
\hline & Capsella rubella & $J G l$ & v1.0 & 81,985 & {$[39,42]$} \\
\hline & Theobroma cacao & $J G l$ & v1.1 & 3641 & {$[39,43]$} \\
\hline \multirow[t]{5}{*}{ Mammalian species } & Canis lupus familiaris & $\mathrm{NCBI}$ & CanFam3.1/AR105 & 6915 & {$[44,45]$} \\
\hline & Homo sapiens & $\operatorname{cCDS}^{3}$ & GRCh38.p7/CCDS20 & 9606 & {$[46]$} \\
\hline & Monodelphis domestica & $\mathrm{NCBI}$ & MonDom5 / AR103 & 13,616 & {$[45,47]$} \\
\hline & Mus musculus & CCDS & GRCm38.p4/CCDS21 & 10,090 & [46] \\
\hline & Oryctolagus cuniculus & $\mathrm{NCBI}$ & OryCun2.0/AR102 & 568,996 & {$[45,48]$} \\
\hline \multirow[t]{5}{*}{ Fungal species } & Eremothecium gossypii & $J G$ & ATCC10895 & 284,811 & [49] \\
\hline & Debaryomyces hansenii & $J G l$ & CBS767 & 284,592 & {$[50,51]$} \\
\hline & Kluyveromyces lactis & $J G l$ & CLIB210 & 284,590 & {$[50]$} \\
\hline & Saccharomyces cerevisiae & $S G D^{4}$ & S288C & 559,292 & [52] \\
\hline & Yarrowia lipolytica & $J G l$ & CLIB122 & 284,591 & [50] \\
\hline
\end{tabular}

JJoint Genome Institute; ${ }^{2}$ National Centre for Biotechnology Information; ${ }^{3}$ Consensus Coding Sequence Project; ${ }^{4}$ Saccharomyces Genome Database

Table 2 SRA RNA-seq data sources

\begin{tabular}{|c|c|c|c|}
\hline & Species & SRA ID & Instrument/details \\
\hline \multirow[t]{5}{*}{ Plants } & A. thaliana & SRR3932355 & Illumina HiSeq 2500, paired end. Wild type Columbia \\
\hline & B. rapa & SRR2984945 & Illumina HiSeq 2000, paired end. ga-deficient dwarf (gad1-2) + GA rep2 \\
\hline & C. papaya & SRR3509576 & Illumina HiSeq 2500, paired end. SunUp/Sunset cultivar, young hermaphrodite leaf \\
\hline & C. rubella & SRR3993756 & Illumina HiSeq 2000, paired end. Leaf sample \\
\hline & T. cacao & SRR3217315 & Illumina HiSeq 2000, paired end. Flower/leaf sample \\
\hline \multirow[t]{13}{*}{ Mammals } & C. lupus & ERR266386 & Illumina Genome Analyzer II, paired end, brain frontal cortex, male \\
\hline & & ERR266355 & Illumina Genome Analyzer II, paired end, brain frontal cortex, female \\
\hline & & ERR266382 & Illumina Genome Analyzer II, paired end, brain frontal cortex, male \\
\hline & H. sapiens & SRR5938455 & Illumina HiSeq 2000, paired end, dorsolateral prefrontal cortex, male \\
\hline & M. domestica & SRR500906 & Illumina HiSeq 2000, paired end, brain \\
\hline & & SRR500925 & Illumina HiSeq 2000, paired end, brain \\
\hline & M. musculus & SRR5441717 & Illumina HiSeq 2000, paired end, brain (striatum) \\
\hline & & SRR6269591 & Illumina NovaSeq 6000, paired end, cerebellum \\
\hline & O. cuniculus & ERR266399 & Illumina Genome Analyzer II, paired end, brain frontal cortex, female \\
\hline & & SRR400990 & Illumina Genome Analyzer II, paired end, brain frontal cortex \\
\hline & & SRR401040 & Illumina Genome Analyzer II, paired end, brain frontal cortex \\
\hline & & SRR401041 & Illumina Genome Analyzer II, paired end, brain frontal cortex \\
\hline & & SRR401042 & Illumina Genome Analyzer II, paired end, brain frontal cortex \\
\hline \multirow[t]{3}{*}{ Fungi } & K. lactis & SRR1200528 & Illumina Genome Analyzer II, single \\
\hline & S. cerevisiae & SRR539284 & Illumina HiSeq 2000, paired end \\
\hline & Y. lipolytica & SRR868669 & Illumina HiSeq 2000, single \\
\hline
\end{tabular}




\section{Multipartite choice function}

The multipartite choice function (Fig. 1) aims, for a set of $k$ gene regions and a set of $l_{k}$ gene model variants for each gene region, to choose an optimal set containing one gene model variant from each gene region such that the alignment score is maximised. This problem is equivalent to finding the heaviest maximal clique in an edge-weighted complete multipartite graph. This function replaces the naïve approach of calculating pairwise alignment scores between all options and choosing an optimal subset, which, while optimal, is computationally infeasible.

To reduce the complexity of the problem, options are chosen by comparison with a reference consensus alignment, produced by taking the most consistent set of amino acids for each column in a global alignment individually (Fig. 1a-b). This column-wise optimisation is fast, and provides a basis for the sequence-wide optimisation. To produce the consensus, The set of $\Sigma l_{k}$ options is aligned to the reference (the original alignment) using MAFFT -add [29]. The inconsistent regions are then isolated and re-aligned using the more accurate but more computationally intensive MAFFT L-INS-i. For each column in the alignment, the set of amino acid choices (one for each gene region) that optimises the alignment score for that column is chosen.
For each option $i$ a binary string $H_{i}=\left\{h_{1}^{i}, \ldots, h_{n}^{i}\right\}$ is produced describing for each position in the alignment whether or not that option matches the consensus (Fig. 1c). The chosen subset will be the set of options that globally maximises agreement with the consensus. If the strings $\left\{H_{i}\right\}_{i}$ are stacked vertically, such that they can be read as columns $\left\{V_{j}\right\}_{j=1}^{n}$ then the task is equivalent to finding a columnar binary string $V$ with one nonzero entry for each gene region such that $\left|V_{i}: V \quad V_{i}\right|$ is maximised.

Given the set $A_{0}=\left\{V_{j}\right\}_{j=1}^{n}$, an optimal subset is deduced by sequential random sampling. Ignoring all-1 strings, an initial $W_{0}=V_{k}$ is chosen at random from $A_{0}$. For sets $S_{1}, S_{2}$ and a set of "checkpoints" $R$, the set $S_{1}$ is compatible with $S_{2}$ with respect to $R=\left\{R_{i}\right\}_{i}$ if the binary intersection $S_{1} \cap S_{2} \cap R_{i}$ is nonzero for all $i$. Define $A_{n}=\left\{a \cap W_{n-1}: a, W_{n-1}\right.$ compatible w. r. t $\left.G\right\}$, where $G$ is the set of binary strings which are zero for all but one gene region, at each stage choosing $W_{n}$ at random from $A_{n}$. The process $A_{0}, A_{1}, A_{2}, \ldots$ eventually converges on a single binary string. This reduction is performed a user-selected number of times, the default being 1000. The result that is a subset of the largest number of $V_{i}$ is declared the winner. In the event that the result still contains more than one option for each gene region, subsets of options are calculated and their multiple alignment score $\alpha$ is calculated, the winner being the subset with the

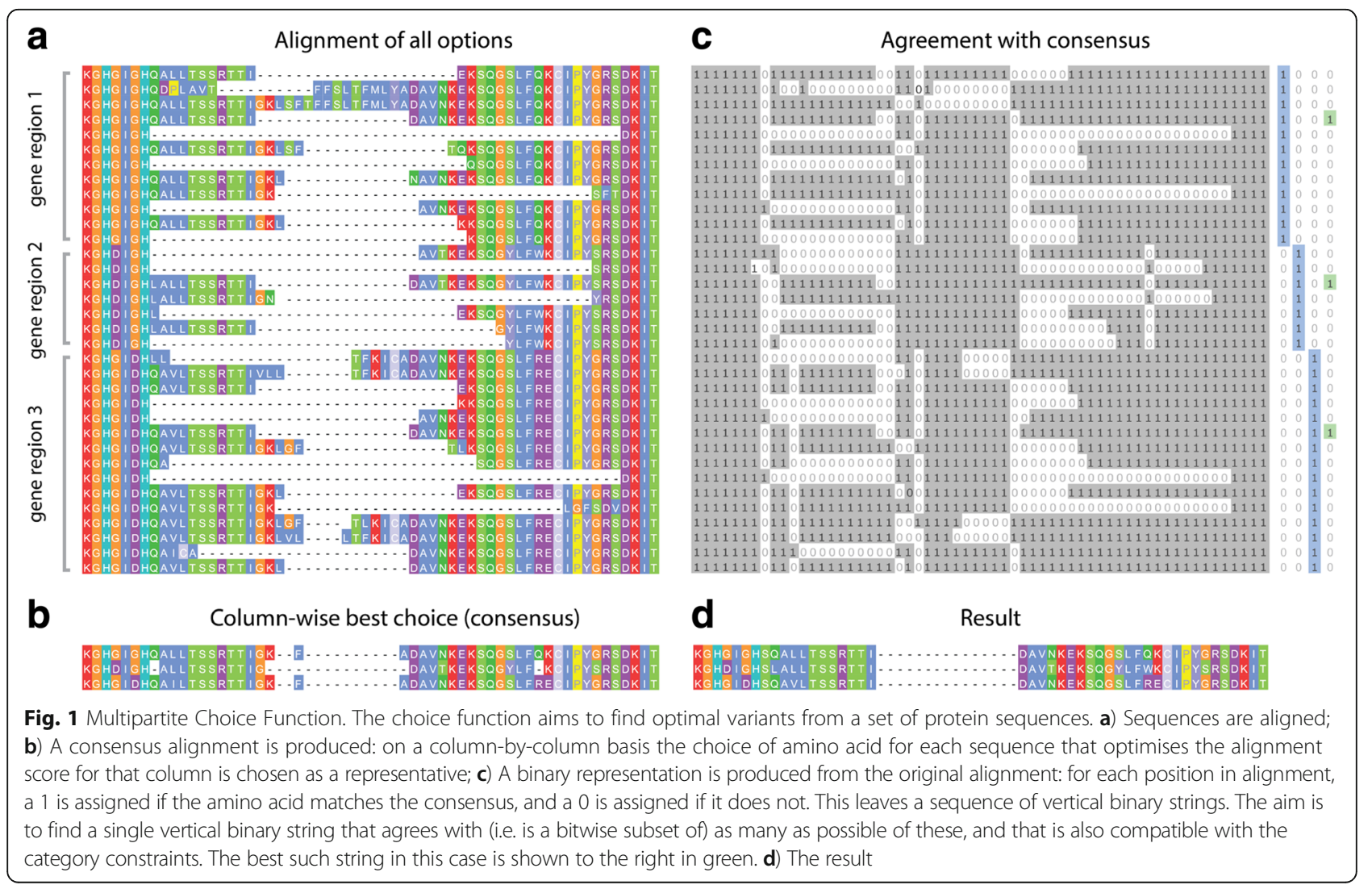


highest $\alpha$. In the event that multiple subsets exhibit the same maximal $\alpha$, a subset is chosen arbitrarily from them.

\section{Adjacency group calculation}

OMGene builds genes sequentially by iteratively adding in putative exons to multiple genes simultaneously. Care must be taken to ensure the gene parts (which in turn become exons once gene models are constructed) are added in a way conducive to vertical comparison of relevant regions (see Fig. 2). In OMGene, gene parts are considered in sequential adjacency groups based on their coordinates in a multiple sequence alignment. Prototype gene models are formed by stringing together amino acid sequences for individual putative exons for each gene region: these are then aligned, and a graph is formed from this alignment. Each putative exon is a node on the graph, and two exons are connected by an edge if and only if one of the exons overlaps the other by a third or more of its length. The adjacency groups are then defined to be cliques in this graph. Cliques are determined using the python implementation of the NetworkX package [30].

\section{Junction F-score}

The junction F-score for a gene is a measure of how well the splice junctions observed in mapped RNA-seq data are represented in the gene model. For a gene model $G$ and corresponding gene region $R$, define $J_{G}$ to be the set of individual intron beginning and end coordinates in the gene model, and define $J_{R}$ to be the set of mapping junction beginning and end coordinates in the mapped RNA-seq data. A minimum of 10 reads is required for a given RNA-seq junction to be counted. We may then define the junction F-score as:

$$
j F\left(J_{G}, J_{R}\right)=\frac{2 \cdot j P\left(J_{G}, J_{R}\right) \cdot j R\left(J_{G}, J_{R}\right)}{j R\left(J_{G}, J_{R}\right)+j P\left(J_{G}, J_{R}\right)}
$$

where

$$
j P\left(J_{G}, J_{R}\right)=\frac{\left|J_{G} \cap J_{R}\right|}{\left|J_{R}\right|} ; \quad j R\left(J_{G}, J_{R}\right)=\frac{\left|J_{G} \cap J_{R}\right|}{\left|J_{G}\right|}
$$

The direction of each junction site (start or end of a junction) is taken into account when considering the intersection of the two sets.

\section{Coverage score}

The coverage score is a measure of how well RNA-seq data represents a given gene. Given that gene expression levels can vary considerably and irregularly across the length of a transcript [13-16], care must be taken to ensure the expression profile for a gene region is properly interpreted. For example, sample preparation methods can bias coverage towards the centre and 3' ends of the transcript; furthermore, jagged read profiles and transcription of antisense regions [31] and other intronic ncRNAs can cause expression profiles to be highly non-binary. To mitigate this, a rolling threshold

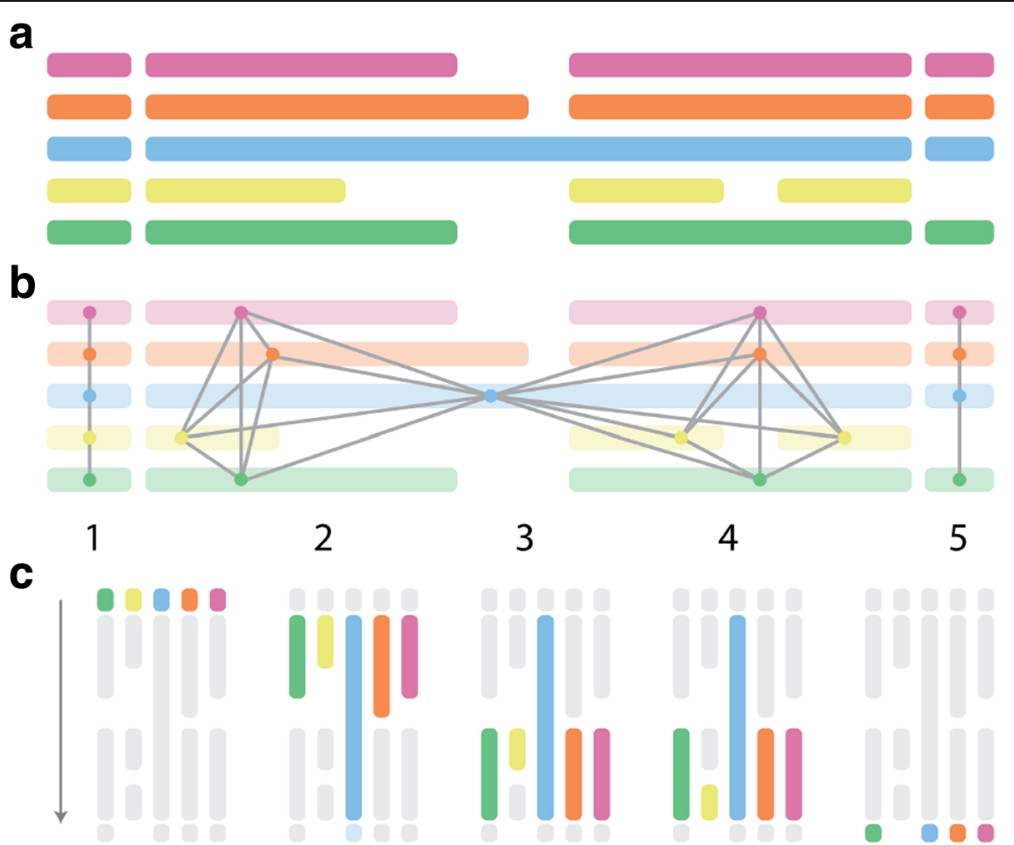

Fig. 2 Calculation of adjacency groups. a) Amino acid sequences for individual putative exons are strung together and aligned. b) A graph is formed with vertices formed by gene parts (or exons), and edges drawn when the overlap between two parts is greater than or equal to one third the length of one of them. c) Cliques are extracted and then ordered lexicographically to form the adjacency groups 
approach is used. For a gene region $R$, and a genomic coordinate $x \in R$, the expression characteristic $X$ is defined as:

$$
\begin{aligned}
\chi(x)= & \min (\max (\{\rho(y): y \in R, y<x\}), \\
& \max (\{\rho(y): y \in R, y>x\}))
\end{aligned}
$$

Where $\rho(y)$ is the read count at genomic coordinate $y$. Bases in the gene region to which the RNA-seq data has been mapped are categorised based on whether they are likely to correspond to exonic or non-exonic regions: a base $x$ is considered to be exonic or on (i.e. likely included in the mature mRNA) if $\rho(x)>\frac{\chi(x)}{5}$, and intronic or off (i.e. likely not included in the mature mRNA) if $\rho$ ( $x)<\frac{X(x)}{5}$. The coverage score for a gene model $G=\left\{G_{1}\right.$, $\left.\ldots, G_{n}\right\}$, where the $G_{i}$ are alternately exons and introns, is defined as:

$$
C(G)=\frac{1}{n}\left(\sum_{G_{i} \text { exonic }} \frac{\mid\left\{x \in G_{i}: x \text { on }\right\} \mid}{\left|G_{i}\right|}+\sum_{G_{j} \text { intronic }} \frac{\mid\left\{x \in G_{i}: x \text { off }\right\} \mid}{\left|G_{j}\right|}\right)
$$

that is, the average length-adjusted coverage score for each individual feature (exon or intron) in the gene model.

\section{RNA-seq data}

RNA-seq data were downloaded from the Sequence Read Archive, assessed using FastQC [32], trimmed using SeqTK [33] and aligned to the genome with $\mathrm{Hi}$ SAT2 [34, 35] using default parameters and single or paired-end methods as appropriate. Per-base coverage was calculated using SAMtools mpileup [36].

\section{Subcellular localisation analysis}

Subcellular localisation predictions for all datasets were obtained using TargetP [37]. For the plant dataset only, TargetP was run with the -P option to predict chloroplast targeting sequences. The localisation consistency for an orthogroup $O$ was calculated as an entropy score across the categories for each gene:

$$
H(O)=-\frac{1}{|O|} \sum_{C \in \mathcal{C}(O)} \frac{|C|}{|O|} \cdot \log \left(\frac{|C|}{|O|}\right)
$$

where $\mathcal{C}(O)=\left\{C_{1}, \ldots, C_{n}\right\}$ is the partition of genes in $O$ into their localisation categories.

\section{Results}

\section{Problem definition, algorithm overview and evaluation} criteria

An overview of the OMGene algorithm is provided in Fig. 3. OMGene aims to find the most consistent set of representative gene models for a set of inputted genes by seeking to maximise the agreement of their aligned amino acid sequences, returning the single best gene model for each gene. The algorithm constructs gene models based on relatively simple constraints: AUG for start codons; GU or GC for splice donor sites, AG for splice acceptor sites, and UAA, UGA, or UAG for stop codons. Other features such as codon bias or polypyrimidine tracts are not considered. OMGene can also use non-canonical translation initiation and splice sites if inputted by the user as a command-line option.

The input for OMGene is a user-selected set of gene models, in general transfer format (GTF), which are assumed to belong to a single orthogroup. For a given set of species, an orthogroup is the set of genes descended from a single ancestral gene in the last common ancestor of those species [26]: this set may contain paralogous as well as orthologous genes. The suggested pipeline for using OMGene is to determine orthogroups using OrthoFinder [26], and to apply OMGene to each or a chosen subset of these orthogroups.

OMGene uses Exonerate [25] as an initial step to cross-align amino acid sequences from all user-supplied genes to the genomic regions of the genes from all species in question, in order to find conserved translatable features. It then combines this information with the original gene models to produce an initial set of prototype exonic regions, or gene parts, for optimisation. The amino acid sequences for these prototype gene models are then aligned, and the constituent gene parts are split into adjacency groups based on overlaps in the alignment (see Implementation). Adjacency groups are sequentially appended to the gene models, and the genetic coordinates are recursively adjusted and assessed to optimise the agreement of the amino acid sequences between species. The resultant gene models are then subject to stringent filtering criteria before the finalised set of gene models are presented as sets of GTF coordinates, amino acid FASTA and coding sequence (CDS) FASTA sequences.

To demonstrate the utility of OMGene, it was applied to orthogroups formed from three sets of test species: a set of five plant species, a set of five mammal species and a set of five fungal species (Table 1). Prokaryotes were not considered, as their protein coding genes lack introns and intergenic regions are either very small or absent and thus gene prediction is comparatively trivial. For plants and fungi, 600 buffer bases either side of each gene model were included, for mammals, which have larger introns, 2000 buffer bases were used. OMGene was applied to orthogroups that contained exactly one gene from each species, referred to as single-copy ubiquitous (SCU) orthogroups. In addition, OMGene was run on the same set but with all genes from three representative species - A. thaliana, H. sapiens and S. cerevisiae - replaced with de novo predicted genes, obtained 


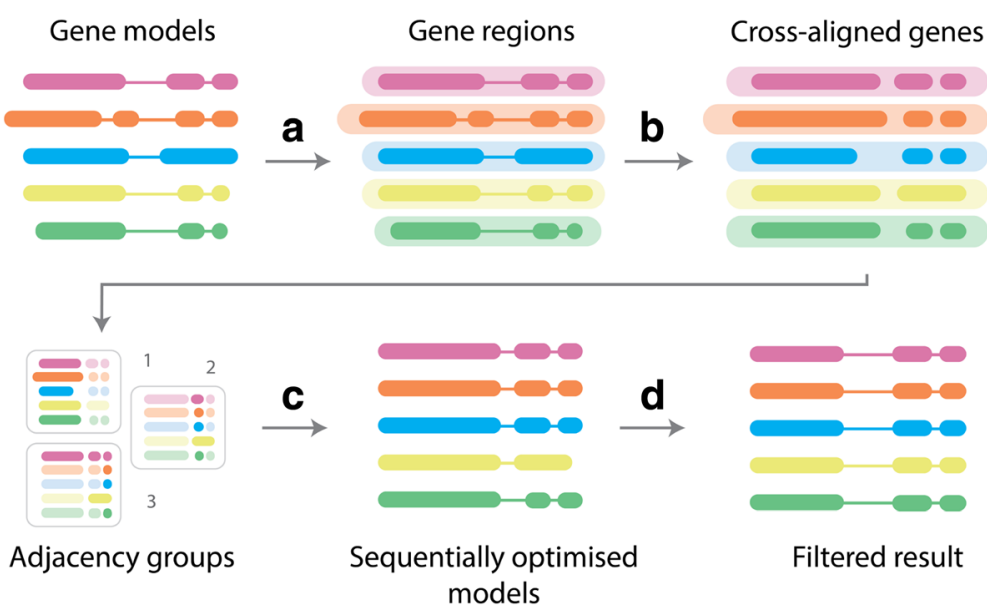

Fig. 3 Simplified overview of OMGene workflow. a) Gene regions are extracted from around the gene model; b) Exonerate is used to cross-align all constituent exons and full open reading frames to construct basic prototype gene models; $\mathbf{c}$ ) The exonic regions from these prototype gene models are sorted into adjacency groups, which are then sequentially optimised using the multipartite choice function;

d) Results are compared against the original gene models to incorporate potentially overlooked combinations, and filtered under various criteria to produce results

by running the Augustus [24] gene finder on those genomes. These species were chosen as they have the best annotated genomes and thus the existing gene models will provide the best possible training set for Augustus de novo prediction. This de novo prediction analysis was done to simulate a typical genome-sequencing project where a user has generated a well-trained set of gene models solely using computational prediction.

OMGene was assessed in three ways: RNA-seq data were used to compare the accuracy of gene models before and after application of OMGene, from both coverage (i.e. the proportion of the predicted gene that is encompassed by reads mapped from RNA-seq data) and splice junction perspectives. To assess the accuracy of start codon prediction, OMGene-modified gene models were subject subcellular localisation prediction and the results were evaluated for consistency across the orthogroup. The RNA-seq data used to assess the success of OMGene were downloaded from the NCBI Sequence Read Archive [28] and are listed in Table 2.

\section{Application of OMGene to publicly available datasets Quantities and nature of changes made}

The full plant data set contained 3694 SCU orthogroups, containing 18,470 genes. Application of OMGene to this test set resulted in gene model changes to one or more genes in $1543(41.8 \%)$ of these orthogroups. In total, 2017 of the inputted genes (10.9\%) were altered. Of these altered versions, 154 genes ( $7.6 \%$ of 2017 ) were present in the original genome annotations as alternative (non-primary) transcripts for the inputted gene. Figure 4 shows examples of various types of gene model alteration for genes in A. thaliana. A full breakdown of per-species change quantities for all species sets can be found in Table 3, Figs. 5 and 6; Table 4 and Fig. 7 show the distribution of the types of changes made. All gene models that were changed by OMGene are included in the supplementary material as a set of GTF files.

The plant species that experienced the highest number of changes were C. papaya and T. cacao, which is consistent with them being more recently published and less well-studied genomes. For all species, more nucleotides were removed than were added, indicating either that gene models predictions tend to be over-cautious or that OMGene is more proficient at removing material than at adding it in. In terms of the types of changes made, exon deletion was by far the most commonly seen change, followed by moved start codon and exon boundary adjustment (Fig. 7). It should be noted that exon deletion events also encapsulate the separation of erroneously fused gene models, which can contribute many exon deletion events simultaneously.

For the mammal data set, $8771 \mathrm{SCU}$ orthogroups were considered, containing 43,855 genes. Of these, 2100 orthogroups (23.9\%) saw some change, with 2686 genes (6.1\%) undergoing alterations. Most gene changes occurred in C. lupus, M. domestica, and O. cuniculus, which, compared to M. musculus and $H$. sapiens, are less well studied and more recently annotated. By far the most common alterations here were removed exons and moved start codons, which is likely partially due to the choice of representative gene model for input to OMGene - the longest.

For the full fungal data set, $2710 \mathrm{SCU}$ orthogroups were considered, containing 13,550 genes. Of these, 100 orthogroups (3.7\%) exhibited some change, and 109 


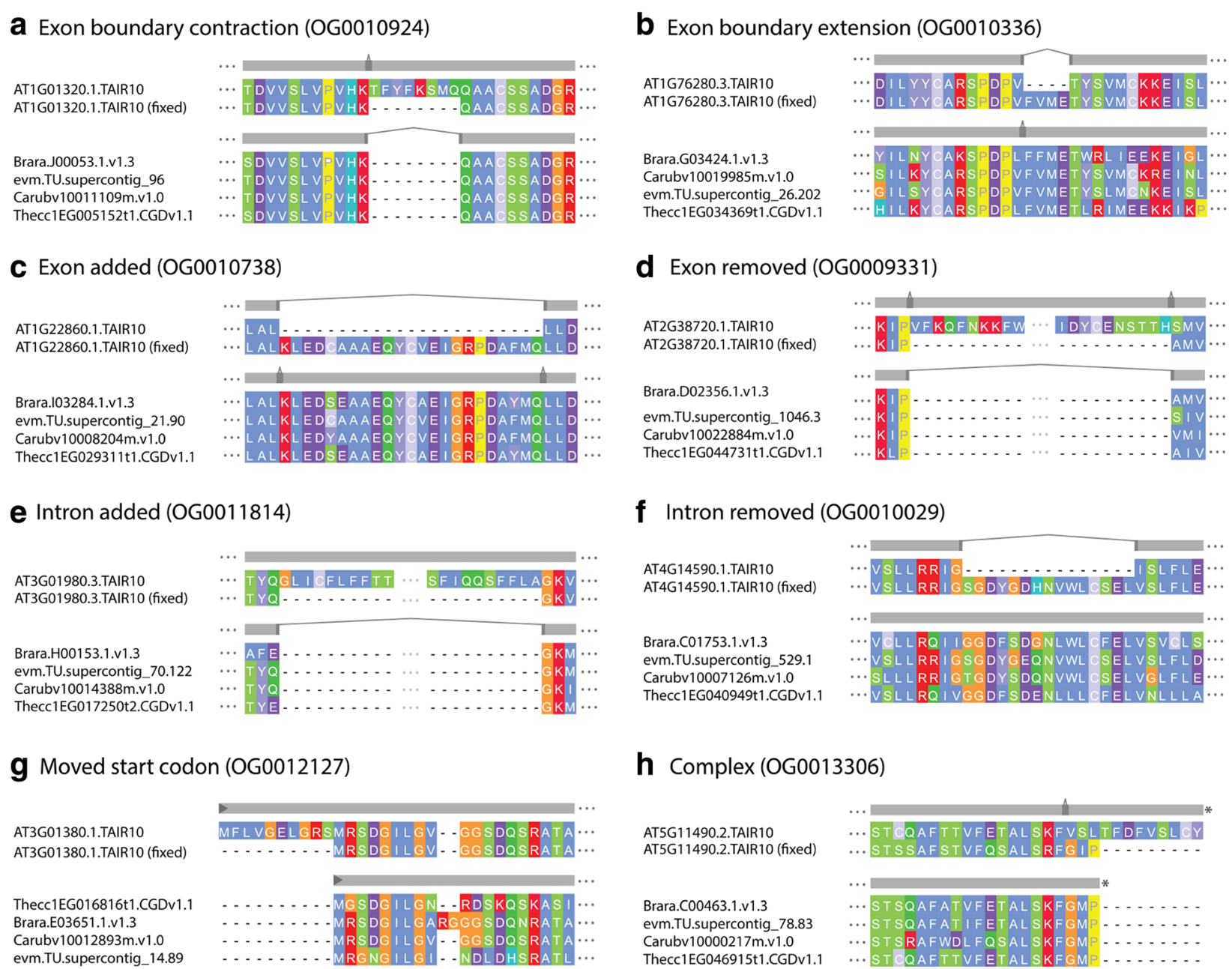

Fig. 4 Examples of individual gene model changes for genes in A. thaliana. a) AT1G01320.1, orthogroup OG0010924, exon extension, splice acceptor side; b) AT1G76280.3.TAIR10, orthogroup OG10336, exon contraction, splice acceptor side; c) AT1G22860.1, orthogroup OG0010738, novel exon introduced; d) AT2G38720.1, orthogroup OG0009331, removed exon; e) AT3G01980.3, orthogroup OG0011814, novel intron introduced; f) AT4G14590.1, orthogroup OG0010029, intron removed; g) AT3G01380.1, orthogroup OG0012127, moved start codon; h) AT5G11490.2, orthogroup OG0013306, complex event: exon has been removed and the previous exon boundary has been extended to include the stop codon

genes $(0.8 \%)$ were altered. In this case, the genes in $E$. gossypii were the most commonly altered, consistent again with it being one of the lesser-studied species on the list. By far the most common change type in the fungal data set was a moved start codon, consistent with the fact that splicing is a rare event in fungal genes (on average 5.09 exons for plants, 11.23 exons for mammals, 1.08 exons for fungi).

To simulate a de novo genome annotation project, OMGene was also applied to the same three data sets with de novo predicted gene models for representative species, A. thaliana, $H$. sapiens and $S$. cerevisiae. These species were chosen as they have the most complete annotations of their respective data sets, and therefore these genes are likely to be the most reliable for training a gene finding algorithm. The genome annotation tool used was Augustus (see Implementation) as it is one of the best and most frequently used gene prediction algorithms.

For the plants data set with Augustus predictions for A. thaliana, 3694 SCU orthogroups were considered. Of these, $598(16.2 \%)$ saw some change in an A. thaliana gene. Similarly, for the mammal data set, 7311 SCU orthogroups were considered, of which 2907 saw changes in a $H$. sapiens gene. For both A. thaliana and $H$. sapiens most changed genes underwent several individual changes. For the fungi data set, $2710 \mathrm{SCU}$ orthogroups were considered. Of these, 19 (0.7\%) saw some change in a S. cerevisiae gene. Tables 3 and 4 show a full breakdown of the types and amounts of changes made. As expected, in both cases, the total number changes and the average size of change made is greater 
Table 3 Per-species gene change breakdown

\begin{tabular}{|c|c|c|c|c|c|c|}
\hline \multirow[t]{2}{*}{ 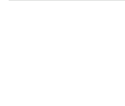 } & \multirow[t]{2}{*}{ Species } & \multirow{2}{*}{$\begin{array}{l}\text { No. changed } \\
\text { genes }\end{array}$} & \multicolumn{3}{|c|}{ Nucleotides added/removed (means per change) } & \multirow{2}{*}{$\begin{array}{l}\text { In original annotation as } \\
\text { alternative "non-primary" } \\
\text { gene model }\end{array}$} \\
\hline & & & $+($ mean $)$ & $+($ mean $)$ & $+($ mean $)$ & \\
\hline \multirow[t]{7}{*}{ Plants } & A. thaliana & 175 & $1749(43)$ & $-23,747(-118)$ & $-22,139(-92)$ & $53(30.3 \%)$ \\
\hline & B. rapa & 97 & $1787(58)$ & $-25,740(-250)$ & $-23,953(-179)$ & $4(4.1 \%)$ \\
\hline & C. papaya & 540 & $23,820(65)$ & $-72,053(-128)$ & $-48,233(-52)$ & $0(0.0 \%)$ \\
\hline & C. rubella & 298 & $6568(71)$ & $-55,005(-170)$ & $-48,437(-117)$ & $2(0.7 \%)$ \\
\hline & T. cacao & 556 & $3700(45)$ & $-120,984(-118)$ & $-117,284(-124)$ & $95(17.1 \%)$ \\
\hline & TOTAL & 1666 & $37,624(61)$ & $-297,529(-145)$ & $-259,905(-97)$ & $154(9.2 \%)$ \\
\hline & A. thaliana de novo & 598 & $13,623(42)$ & $-167,038(-35)$ & $-51,177(-57)$ & N/A \\
\hline \multirow[t]{7}{*}{ Mammals } & C. lupus & 698 & $8993(64)$ & $-98,467(-117)$ & $-89,474(-91)$ & 375 (53.7\%) \\
\hline & H. sapiens & 397 & $4429(59)$ & $-41,401(-101)$ & $-36,972(-76)$ & $218(54.9 \%)$ \\
\hline & M. domestica & 787 & $8637(53)$ & $-100,256(-101)$ & $-91,619(-80)$ & 349 (44.3\%) \\
\hline & M. musculus & 270 & $9685(120)$ & $-19,236(-79)$ & $-9551(-29)$ & 81 (30.0\%) \\
\hline & O. cuniculus & 534 & $12,038(61)$ & $-72,398(-112)$ & $-60,360(-71)$ & $243(45.5 \%)$ \\
\hline & TOTAL & 2686 & $43,782(67)$ & $-331,758(-106)$ & $-287,976(-76)$ & $1266(47.1 \%)$ \\
\hline & H. sapiens de novo & 2907 & $251,344(79)$ & $-952,864(-167)$ & $-701,520(-79)$ & N/A \\
\hline \multirow[t]{7}{*}{ Fungi } & E. gossypii & 46 & $0(0)$ & $-4338(-93)$ & $-4338(-93)$ & N/A \\
\hline & D. hansenii & 13 & $0(0)$ & $-2080(-149)$ & $-2080(-149)$ & N/A \\
\hline & K. lactis & 11 & $0(0)$ & $-1314(-110)$ & $-1314(-110)$ & N/A \\
\hline & S. cerevisiae & 11 & $93(93)$ & $-2483(-191)$ & $-2390(-170)$ & $\mathrm{N} / \mathrm{A}$ \\
\hline & Y. lipolytica & 23 & $117(29)$ & $-4186(-199)$ & $-4069(-163)$ & N/A \\
\hline & TOTAL & 104 & $210(42)$ & $-14,401(-135)$ & $-14,191(-127)$ & N/A \\
\hline & S. cerevisiae de novo & 19 & $601(120)$ & $-5561(-347)$ & $-4960(-236)$ & N/A \\
\hline
\end{tabular}

for the de novo predicted gene models than the curated gene models. However, the distribution of types of changes made remained roughly the same.

\section{Splice junction and feature coverage analysis}

To assess the validity of changes made by OMGene, both the original and the updated gene model sets were compared using publicly available RNA-seq data from the NCBI Sequence Read Archive [28] (see Implementation and Table 2). Data were downloaded for all species except E. gossypii and D. hansenii, for which no adequate data were available. Each amended gene was assessed in two ways relative to this data: firstly by comparing the exact splice junction locations with RNA-seq derived splice junctions; secondly by evaluating the coverage of exonic regions with RNA-seq. To control for unreliable data, some genic regions were omitted from this analysis. Gene regions in which the RNA-seq data suggested there were indels in the reference genome, or that were within $1000 \mathrm{bp}$ of the end of a contig or scaffold, or that contained 10 or more contiguous " $\mathrm{N}$ " nucleotide bases were omitted from the analysis (see Implementation). Regions with these characteristics prevent the creation of reliable gene models, and so are deemed here "unassessable" and not useful for determining gene model accuracy. Gene regions with very low coverage were also omitted from the junction score analysis: gene regions required at least one base with a read depth of 10 or more.

Gene models outputted by OMGene were assessed on whether or not their junction and coverage F-scores (see Implementation) had improved or been reduced. The full results can be seen in Table 5. For the plant data set, OMGene improved the agreement of the gene model with the splice junctions inferred from RNA-seq data for 729 assessable genes, while 125 assessable gene models exhibited reduced agreement (85.3\% improved). Similarly, when assessing RNA-seq coverage of gene models OMGene improved the agreement of the models with the data for 1026 genes, while 167 genes exhibited reduced agreement (86.0\% improved). For the de novo predicted $A$. thaliana genes, the success rates were essentially the same as for the public data $(87.3 \%$ and 91.1\% improved by junction and coverage F-scores respectively), but the absolute quantity of genes exhibiting a changed score increased roughly four-fold. This difference represents the considerable effort and evidence-based curation that has been invested in the A. thaliana genome annotation.

For the mammals, 948 assessable changed genes (79.2\%) had an improved junction score compared to the original 


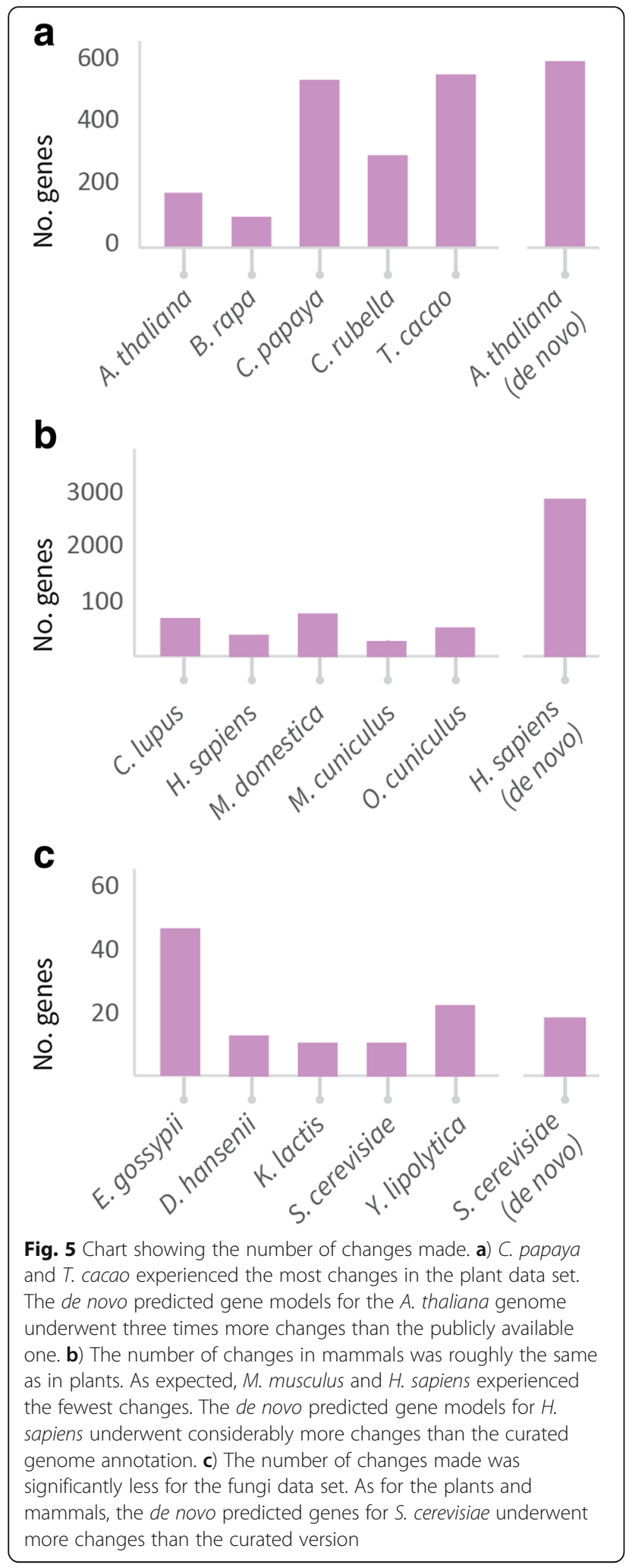

gene model. In addition, $82.83 \%$ of assessable genes had improved RNA-seq coverage scores. For the de novo data, $89.4 \%$ of assessable predicted $H$. sapiens had an improved

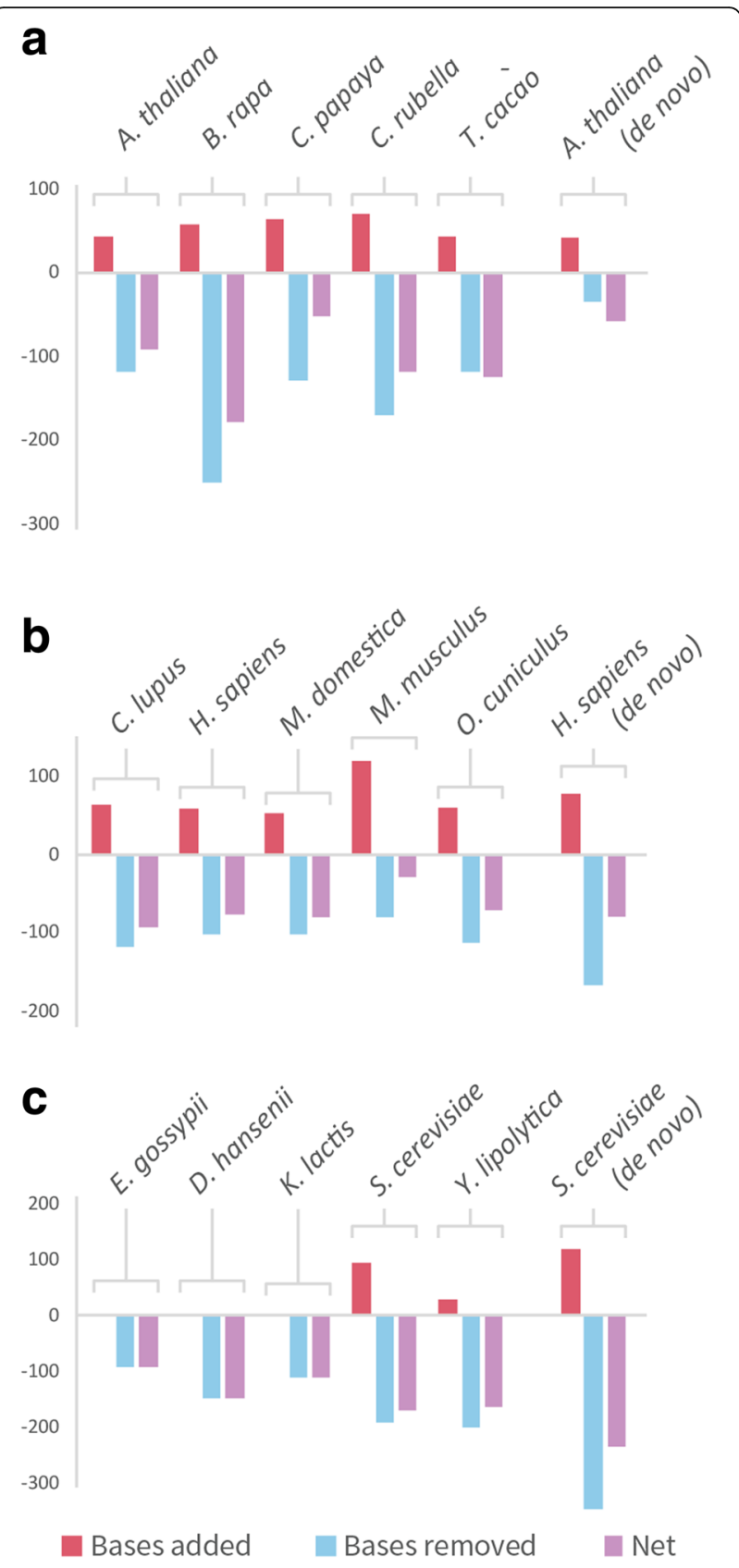

Fig. 6 The average number of nucleotides added or removed from gene models as a result of changes made by OMGene. The units of the $y$-axis are the number of nucleotides. a) Average magnitudes of each change for plants; $\mathbf{b})$ Average magnitudes for changes made to mammal genes; c) Average magnitudes for changes made to fungal genes. In all cases, predicted gene sequences were shortened to a greater extent than they were lengthened.

splice junction score after application of OMGene, and $88.74 \%$ had an improved coverage score. The numbers of changed genes were again considerably more for the de novo predicted $H$. sapiens data, again indicating the high degree of attention that has been afforded to the annotation of this species. 
Table 4 Summary of gene model change categories

\begin{tabular}{|c|c|c|c|c|c|c|c|c|c|}
\hline & \multirow[t]{2}{*}{ Species } & \multirow{2}{*}{$\begin{array}{l}\text { No. } \\
\text { changes }\end{array}$} & \multicolumn{2}{|c|}{ Exon boundary } & \multicolumn{2}{|l|}{ Exon } & \multicolumn{2}{|c|}{ Intron } & \multirow[t]{2}{*}{ Moved star } \\
\hline & & & contraction & extension & add & del & add & del & \\
\hline \multirow[t]{7}{*}{ Plants } & A. thaliana & 242 & 47 & 23 & 4 & 117 & 5 & 13 & 33 \\
\hline & B. rapa & 134 & 11 & 14 & 9 & 56 & 3 & 8 & 33 \\
\hline & C. papaya & 928 & 148 & 205 & 95 & 345 & 18 & 42 & 74 \\
\hline & C. rubella & 415 & 32 & 32 & 39 & 101 & 1 & 19 & 191 \\
\hline & T. cacao & 949 & 117 & 59 & 9 & 624 & 10 & 13 & 117 \\
\hline & TOTAL & 2668 & 355 & 333 & 156 & 1243 & 37 & 95 & 448 \\
\hline & A. thaliana de novo & 1344 & 151 & 255 & 49 & 780 & 2 & 10 & 97 \\
\hline \multirow[t]{7}{*}{ Mammals } & C. lupus & 980 & 116 & 72 & 38 & 418 & 9 & 4 & 323 \\
\hline & H. sapiens & 485 & 31 & 43 & 29 & 223 & 0 & 0 & 159 \\
\hline & M. domestica & 1148 & 122 & 89 & 56 & 472 & 19 & 3 & 387 \\
\hline & M. musculus & 324 & 20 & 29 & 42 & 111 & 6 & 6 & 110 \\
\hline & O. cuniculus & 834 & 139 & 122 & 43 & 245 & 18 & 9 & 258 \\
\hline & TOTAL & 3771 & 428 & 355 & 208 & 1469 & 52 & 22 & 1237 \\
\hline & H. sapiens de novo & 8883 & 606 & 1128 & 1866 & 4217 & 16 & 17 & 1033 \\
\hline \multirow[t]{7}{*}{ Fungi } & E. gossypii & 46 & 0 & 0 & 0 & 1 & 0 & 0 & 45 \\
\hline & D. hansenii & 13 & 0 & 0 & 0 & 1 & 0 & 0 & 12 \\
\hline & K. lactis & 11 & 0 & 0 & 0 & 0 & 0 & 0 & 11 \\
\hline & S. cerevisiae & 13 & 1 & 0 & 0 & 0 & 1 & 1 & 10 \\
\hline & Y. lipolytica & 24 & 0 & 0 & 0 & 4 & 5 & 0 & 15 \\
\hline & TOTAL & 107 & 1 & 0 & 0 & 6 & 6 & 1 & 93 \\
\hline & S. cerevisiae de novo & 20 & 0 & 2 & 0 & 4 & 0 & 2 & 12 \\
\hline
\end{tabular}

The results for the fungal data set (see Table 6) were not as good. Notably very few gene models showed any change in junction F-score, with only 8 genes exhibiting a changed score. This is due to the relatively simple exon structure of fungal genes, for which splicing is very rare, and splicing events predicted by OMGene are much less likely to be correct. In this case 3 genes had an improved score, and 5 had a reduced score (37.5\% success), with all 5 of the losing genes coming from $Y$. lipolytica. The most common change made to fungal genes was a moved start codon, which, although not detectable in the junction F-score, can be detectable in the coverage F-score. This is reflected in the results, where 30 genes showed an improved coverage F-score and 10 genes showed a worse coverage F-score ( $75 \%$ improved). In the de novo case, again the numbers increased while the percentage success remained roughly the same, with 4 (100\%) genes improving by junction for S. cerevisiae and 11 (64.7\%) improving by coverage score. The highly compact nature of fungal genomes, with few exons and limited space between genes means that the accuracy of de novo predicted genes is higher than in plants and mammals. Thus the utility of OMGene on these comparatively simpler genomes is limited.
Many of the cases for which OMGene results differ from RNA-seq evidence are attributable to real biological variability that confounds the evaluation criteria of the algorithm. For example, there are some instances where the most evolutionary conserved splice site was not the splice site observed in the RNA-seq data. Such events, by definition, cannot be detected by OMGene. Furthermore, RNA-seq mapping errors also contributed to reduced scores, as did artefacts resulting from spliced UTRs, and jagged read profiles, particularly in the fungal data, that made some coverage scores difficult to calculate reliably. Finally, the presence of multiple transcript isoforms within the RNA-seq data can reduce the score for a valid transcript even if it is the best choice for that particular gene. While users of OMGene should be aware of these confounding factors, the above data demonstrates that, in general, OMGene is much more likely to improve a given gene model than not even for extensilvey curated genomes.

\section{Assessment of subcellular localisation predictions for 5 ' end analysis}

Given that genes from the same orthogroup are, by definition, descended from a single ancestral gene, it is 


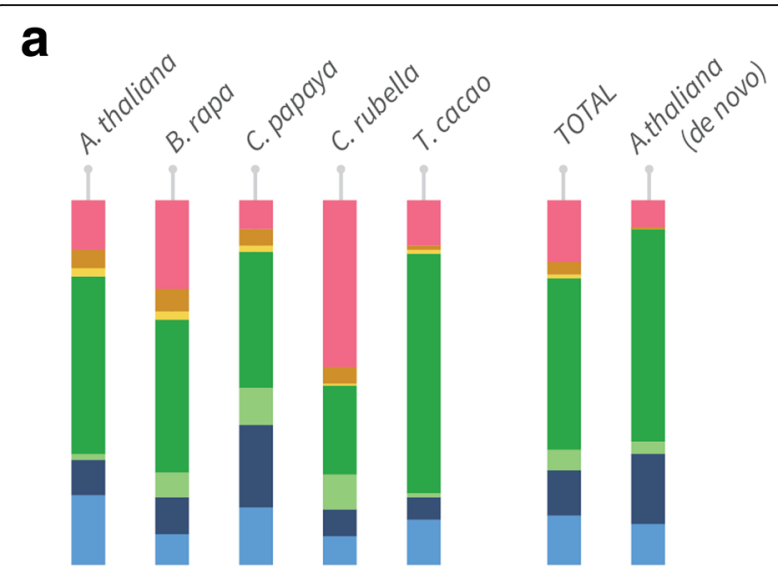

b

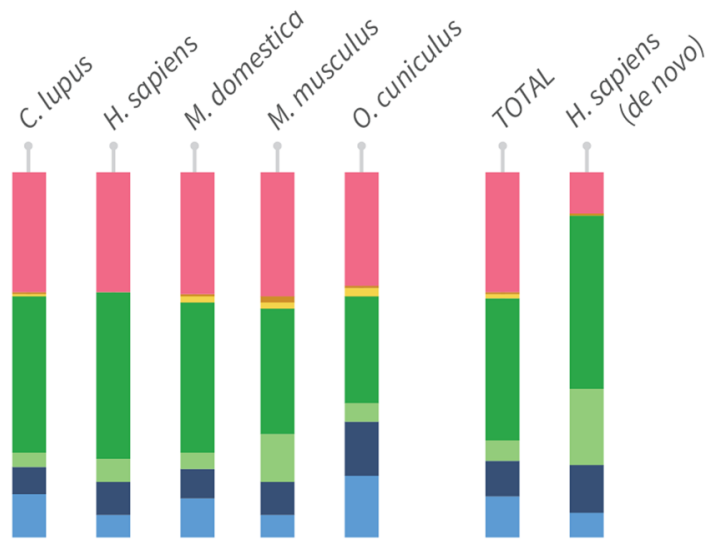

C

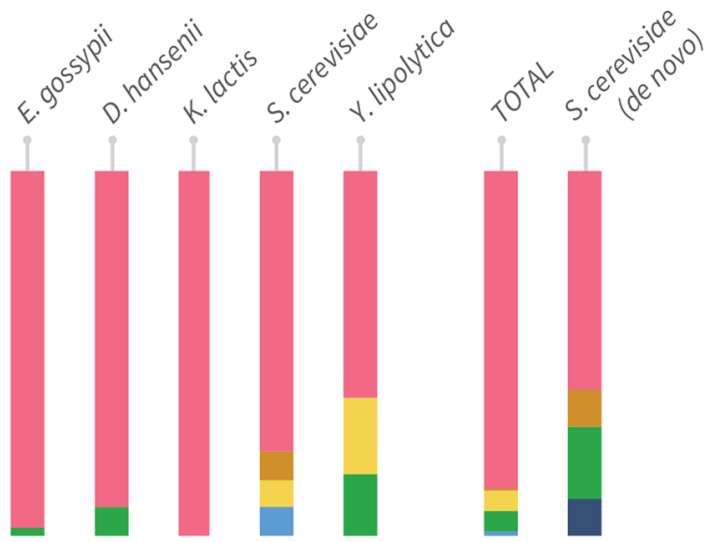

$$
\begin{aligned}
& \text { Exon contraction } \quad \text { Exon addition } \quad \text { Intron addition } \\
& \text { Exon extension } \quad \text { Exon deletion } \quad \text { Intron deletion } \\
& \text { Moved start }
\end{aligned}
$$

Fig. 7 Distribution of types of changes made in the three data sets. a) The most common change in plants was exon deletion. b) Moved start codons and removed exons were most common in mammals. c) In fungi, the most common change was overwhelmingly a moved start codon reasonable to assume that they should be consistent in their predicted subcellular localisation. Several subcellular targeting sequences are located at the $\mathrm{N}$-termini of genes [38], thus one expects genes with inaccurately predicted start codons to yield inaccurate results when assessing their targeting signals. Genes belonging to orthogroups changed by OMGene were assessed to determine whether the changes resulted in increased consistency of the predicted subcellular localisation of all genes in the orthogroup. Targeting predictions were made using TargetP [37], and Shannon entropy was calculated to assess the consistency of the predictions within the orthogroups (see Implementation). Entropy scores were compared only for orthogroups in which at least one gene model was altered by OMGene. An entropy score of 0 indicates that all members of the orthogroup are predicted to localise to the same sub-cellular compartment; the worst possible entropy score given five genes and four possible localisations identified by TargetP (chloroplast, mitochondrion, secreted, cytoplasmic) is $-\frac{2}{5} \log _{2}\left(\frac{1}{5}\right)-\frac{3}{5}$ $\log _{2}\left(\frac{1}{5}\right) \approx 1.92$, indicating that only two of the genes agree. An example orthogroup whose prediction entropy score has been improved by start codon adjustment can be seen in Fig. 8.

The 1543 plant orthogroups in which one or more genes were altered were subjected to subcellular prediction analysis (Table 6). Of these, gene model changes made by OMGene resulted in changes in predicted subcellular localisation for one or more constituent members of 55 orthogroups. In total, 74 improved agreement between gene models (74\%), 13 remained the same (13\%), and 13\% increased disagreement between predicted subcellular localisation of gene models. The effect was more profound for the mammal data: 509 orthogroups showed a change in localisation predictions for one or more of their genes, 444 of which (87.2\%) resulted in improved predicted localisation consistency. The data for fungi were sparser: only 7 out of 95 changed orthogroups exhibited a change in subcellular localisation prediction, with 6 of these changes improving the consistency of localisation prediction (85.7\%) and 1 increasing disagreement (14.3\%).

Similar results were obtained for the de novo annotation analysis in plants, although again the data were sparse here. Orthogroups containing the de novo predicted $A$. thaliana genes were considered together with the four original genes for the other species. Here, 11 of the $A$. thaliana genes experienced a change in subcellular localisation following application of OMGene. Of the 11 orthogroups containing these, 9 improved consistency $(81.9 \%)$ and 2 reduced the consistency (18.2\%). For de novo H. sapiens predictions, 527 of the start codon changes resulted in a change in subcellular localisation prediction change for $H$. sapiens. In 458 (86.9\%) of these cases, this improved the consistency of 
Table 5 RNA-seq coverage and junction F-scores

\begin{tabular}{|c|c|c|c|c|c|}
\hline & \multirow[t]{2}{*}{ Species } & \multicolumn{2}{|c|}{ Junction F-score } & \multicolumn{2}{|c|}{ Coverage F-score } \\
\hline & & Better & Worse & Better & Worse \\
\hline \multirow[t]{7}{*}{ Plants } & A. thaliana & $94(87.8 \%)$ & $13(12.1 \%)$ & $109(91.5 \%)$ & $10(8.4 \%)$ \\
\hline & B. rapa & $24(63.1 \%)$ & $14(36.8 \%)$ & 29 (56.8\%) & $22(43.1 \%)$ \\
\hline & C. papaya & $246(82.2 \%)$ & $53(17.7 \%)$ & $344(83.9 \%)$ & 66 (16.0\%) \\
\hline & C. rubella & 90 (89.1\%) & $11(10.8 \%)$ & $186(91.6 \%)$ & $17(8.3 \%)$ \\
\hline & T. cacao & 275 (88.9\%) & $34(11.0 \%)$ & $358(87.3 \%)$ & $52(12.6 \%)$ \\
\hline & TOTAL & 729 (85.3\%) & $125(14.6 \%)$ & 1026 (86.0\%) & 167 (13.9\%) \\
\hline & A. thaliana de novo & $422(87.3 \%)$ & $61(12.6 \%)$ & $475(91.1 \%)$ & $46(8.8 \%)$ \\
\hline \multirow[t]{7}{*}{ Mammals } & C. lupus & $323(85.9 \%)$ & $53(14.1 \%)$ & 478 (86.28\%) & $76(13.72 \%)$ \\
\hline & H. sapiens & $102(82.9 \%)$ & $21(17.1 \%)$ & $258(87.46 \%)$ & 37 (12.54\%) \\
\hline & M. domestica & $239(72.2 \%)$ & $92(27.8 \%)$ & 439 (76.48\%) & $135(23.52 \%)$ \\
\hline & M. musculus & 71 (62.3\%) & $43(37.7 \%)$ & $133(71.12 \%)$ & $54(28.88 \%)$ \\
\hline & O. cuniculus & $213(84.2 \%)$ & $40(15.8 \%)$ & 353 (86.10\%) & 57 (13.90\%) \\
\hline & TOTAL & $948(79.2 \%)$ & 249 (112.5\%) & $1661(82.83 \%)$ & $359(17.77 \%)$ \\
\hline & H. sapiens de novo & $832(89.4 \%)$ & 99 (10.6\%) & 2017 (88.74\%) & $256(11.26 \%)$ \\
\hline \multirow[t]{5}{*}{ Fungi } & K. lactis & $0(\mathrm{~N} / \mathrm{A})$ & $0(\mathrm{~N} / \mathrm{A})$ & 9 (100.0\%) & $0(0 \%)$ \\
\hline & S. cerevisiae & $0(N / A)$ & $0(N / A)$ & $6(75.0 \%)$ & $2(25.0 \%)$ \\
\hline & Y. lipolytica & $2(28.5 \%)$ & $5(71.4 \%)$ & $11(64.7 \%)$ & $6(35.2 \%)$ \\
\hline & TOTAL & $3(37.5 \%)$ & $5(62.5 \%)$ & $30(75.0 \%)$ & $10(25.0 \%)$ \\
\hline & S. cerevisiae de novo & $4(100 \%)$ & $0(0 \%)$ & $11(64.7 \%)$ & $6(35.2 \%)$ \\
\hline
\end{tabular}

the orthogroup. For the fungal data set, the data were extremely sparse, with only one gene experiencing a change in its targeting prediction, which reduced the consistency for its orthogroup. Thus, although data were sparse for the fungal dataset, in each of the plant, mammal, and fungal datasets the consistency of gene models was on the whole improved from a subcellular targeting perspective.

\section{Discussion}

Here we present OMGene, an automated method for improving the consistency of gene model annotations across species. OMGene is intended for use in computational de novo genome annotation projects where no empirical data (such as RNA-seq data) is available to train or correct gene model predictions, or to assist the construction of gene models for genes that are not expressed in the data available. OMGene is also designed to help users who wish to leverage conservation information to correct gene models of a single gene of interest across a set of species. Thus OMGene is suitable for both large and small scale analyses.

OMGene is run as a python script with a tabdelimited input file, each line of which contains the path to a GTF file, containing coordinates for a single gene, and the path to its corresponding genome FASTA file. Full instructions for running OMGene can be found at the OMGene GitHub repository (https://github.com/ mpdunne/omgene).

Table 6 Subcellular localisation predictions

\begin{tabular}{|c|c|c|c|c|c|}
\hline & \multirow[t]{2}{*}{ Category } & \multirow{2}{*}{$\begin{array}{l}\text { No. orthogroups with } \\
\text { changed localisation } \\
\text { predictions }\end{array}$} & \multicolumn{3}{|c|}{ Entropy score } \\
\hline & & & Better & Same & Worse \\
\hline \multirow[t]{2}{*}{ Plants } & Public data & 55 & $42(76.4 \%)$ & $5(7.7 \%)$ & $8(14.5 \%)$ \\
\hline & A. thaliana de novo & 11 & 9 (81.9\%) & $0(0 \%)$ & $2(18.2 \%)$ \\
\hline \multirow[t]{2}{*}{ Mammals } & Public data & 509 & 444 (87.2\%) & 19 (3.7\%) & $46(9.0 \%)$ \\
\hline & H. sapiens de novo & 527 & 458 (86.9\%) & $23(4.4 \%)$ & $46(8.7 \%)$ \\
\hline \multirow[t]{2}{*}{ Fungi } & Public data & 7 & $6(85.7 \%)$ & $0(0 \%)$ & $1(14.3 \%)$ \\
\hline & S. cerevisiae de novo & 1 & $0(0 \%)$ & $0(0 \%)$ & $1(100 \%)$ \\
\hline
\end{tabular}



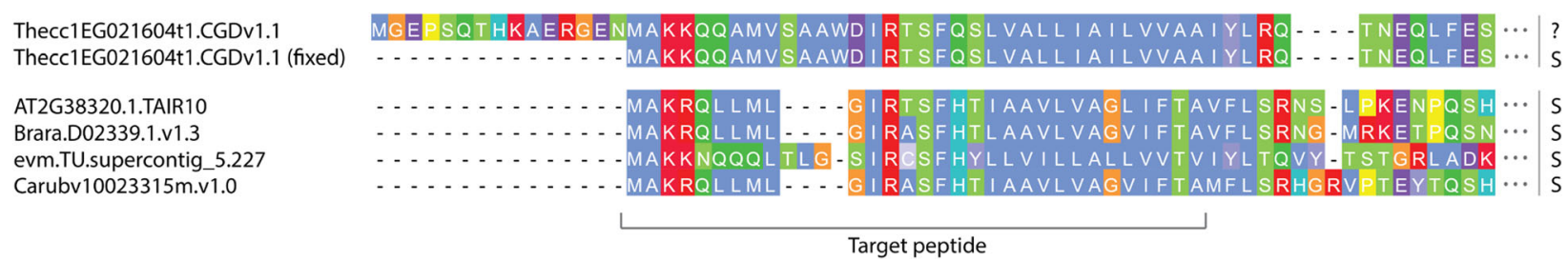

Fig. 8 Example change in subcellular localisation prediction for a gene. Thecc1EG021604t1.CGDv1.1 from T. cacao has undergone a change in start codon, revealing a signal peptide at its $5^{\prime}$ end. In this case, what was previously assumed to be cytosolic has been found to be targeted to the secretory pathway, the same as the other members of the orthogroup (OG0009265). In this case, the Shannon entropy score for the orthogroup has fallen from 0.72 to 0

\section{OMGene results reflect differences in gene model complexity between species sets}

To demonstrate the utility and performance characteristics of OMGene, it was applied to three separate datasets of well-annotated plant, mammal and fungal genomes. When applied to the plant data set, OMGene altered the gene models of one or more genes in $41.8 \%$ of the orthogroups that were evaluated; when applied to the mammal data set, $23.9 \%$ of SCU orthogroups saw some change. In contrast, only $3.7 \%$ of orthogroups were subject to modification in the fungal data set. This result reflects the differences in gene model complexity between the three species groups. Specifically, gene models in plants tend to have more exons than fungi (mean $=5.09$ exons for plants, 11.23 for mammals, 1.08 exons for fungi) and thus there is considerably more potential for gene model variation in plants and mammals than in fungi. In light of this it was unsurprising that the most frequently observed change made in fungi was a change in choice of start codon. This is also reflected in the high number of removed exons from plant and mammal genes, which is contributed to partly by the separation of erroneously fused adjacent genes.

\section{OMGene works well on complex gene models}

The changes made by OMGene were assessed relative to splice-mapped RNA-seq data to assess the level to which it had improved the gene models. For the plant data set, the results from OMGene clearly resembled the empirical data more closely, with $85.4 \%$ and $86.0 \%$ of genes improving in terms of their splice junctions and their coverage respectively. The profiles were different for different species, with many more changes being made for C. papaya and T.cacao; in addition the number of successes for $B$. rapa was slightly lower than for the other species.

In mammals, the three non-model species, C. lupus, $M$. domestica, and O. cuniculus faired well in terms of their junction scores. $H$. sapiens genes also typically scored highly, but $M$. musculus genes did not do so well.
The reason why mouse genes changes were less successful than human gene changes is unknown. These two species also saw considerably fewer gene model changes than the other species. In all cases, mammal genes were mostly contracted via the removal of exons or by moving the start codon - this is at least partly due to biases in the choice of longest gene model as representatives for these genes.

The number of junction changes made for the fungal data set was considerably lower: only 8 changed genes had an altered junction F-score, $62.5 \%$ of which become worse after OMGene. Though this is less than the plant data set, it should be noted that the resolution of this data set does not lend itself to accurate conclusions about the general validity of changes made to fungal genes. The resolution and success rate for fungal genes from a coverage perspective was slightly higher, with $75 \%$ of the genes with changed scores improving. The low resolution of junction data for fungal genes reflects the rarity of complex gene models in these species, and thus the low likelihood that deviations from simple, single-exon gene models are correct. Thus, while OMGene does not always produce gene models that agree with transcriptome data, it does improve the overall quality of gene model annotations even for relatively simple fungal genomes.

The improvements in gene model accuracy made by OMGene for the de novo predicted gene models were much the same as for the publicly available, curated gene models. However, the number of changes made to the de novo predicted set was much greater, indicating that the considerable labour that has been applied to these model organisms has successfully controlled for potential errors. It should be noted that, although OMGene managed to improve many of the gene models outputted by Augustus, the two agreed in most cases $(86.1 \%, 60.3 \%$ and $98.6 \%$ for plants, mammals and fungi respectively), indicating that the basic implementation of a well-trained Augustus de novo prediction produces genes that are highly consistent between species. 


\section{OMGene improves the consistency of subcellular localisation predictions}

In addition to assessment of coverage and splice junctions, gene models were assessed by the consistency of their predicted subcellular localisation. Given that the orthogroups used in this analysis comprise ubiquitously conserved single copy genes, it is logical to assume that these genes should generally have the same subcellular localisation. For the full plant data set, of all orthogroups whose genes had different subcellular targeting predictions after application of OMGene, $76.4 \%$ had improved intra-orthogroup consistency, with $85.5 \%$ either improving or remaining the same. In mammals, $87.2 \%$ of orthogroups whose subcellular localisation predictions were changed by OMGene showed improved consistency. For the full fungal data set, although the data were sparse, $85.7 \%$ of the orthogroups considered had improved consistency.

The results for the plant data set were similar for the de novo annotated set (85.7\% improvement), as was also the case for mammals (86.9\% improvement). For fungal orthogroups containing de novo predicted $S$. cerevisiae genes, the only gene whose localisation prediction changed caused the consistency of its orthogroup to decrease, however the resolution of the data in this case is not sufficient to draw any conclusions. Thus, application of OMGene improves the accuracy of start codon specification in de novo predicted gene models.

\section{Conclusions}

When applied to publicly available plant, mammal and fungal data sets, OMGene demonstrates proficiency in improving gene models from multiple perspectives. Due to stringent filtering criteria, it does not fix all errant gene models, however the gene models that it does fix represent an improvement the majority of the time. The overall improvement is larger for genomes with complex gene models.

\section{Availability and requirements}

Project name: OMGene.

Project home page: https://github.com/mpdunne/omgene

Operating system: Unix.

Programming languages: Python v2.7.x.

Other requirements: Bedtools, Exonerate, Python packages (scipy, numpy, pyfaidx, networkx, BioPython).

License: GPLv3.

\section{Abbreviations}

CDS: Coding sequence; GTF: General transfer format; SCU: Single copy, ubiquitous

\section{Funding}

SK is a Royal Society University Research Fellow. This work was supported by the European Union's Horizon 2020 research and innovation programme under grant agreement number 637765. MPD is supported by an EPSRC studentship through EP/G03706X/1.

\section{Availability of data and materials}

The software is available under the GPLv3 licence at https://github.com/ mpdunne/omgene.

\section{Authors' contributions}

SK conceived the project. MPD developed the algorithm. SK and MPD analysed the data and wrote the manuscript. Both authors read and approved the final manuscript.

\section{Competing interests}

The authors declare that they have no competing interests.

\section{Publisher's Note}

Springer Nature remains neutral with regard to jurisdictional claims in published maps and institutional affiliations.

Received: 17 November 2017 Accepted: 19 April 2018

Published online: 27 April 2018

\section{References}

1. Cochrane G, Karsch-mizrachi I, Nakamura Y. The international nucleotide sequence database collaboration the international nucleotide sequence database collaboration. Nucleic Acids Res. 2011;39:D15-8.

2. Land M, Hauser L, Jun S, Nookaew I, Leuze MR, Ahn T, Karpinets T, Lund O, Kora G, Wassenaar T, Poudel S, Ussery DW. Insights from 20 years of bacterial genome sequencing. Funct Integr Genomics. 2015;15:141-61.

3. NCBI, "GenBank and WGS Statistics," 2017. [Online]. Available: https://www. ncbi.nlm.nih.gov/genbank/statistics/.

4. Hayden EC. The $\$ 1,000$ genome. Nature. 2014;507:295

5. K. Wetterstrand, "DNA Sequencing Costs: Data from the NHGRI Genome Sequencing Program (GSP)," Mar-2016. [Online]. Available: www.genome. gov/sequencingcosts.

6. M. Yandell and D. Ence, "A beginner's guide to eukaryotic genome annotation," Nat Rev Genet, vol 13, no May, pp. 329-342, 2012.

7. Denton JF, Lugo-Martinez J, Tucker AE, Schrider DR, Warren WC, Hahn MW. Extensive error in the number of genes inferred from draft genome assemblies. PLoS Comput Biol. 2014;10(12)

8. E. Veeckman, T. Ruttink, and K. Vandepoele, "Are We There Yet ? Reliably Estimating the Completeness of Plant Genome Sequences," Plant Cell, vol. 28, no. August, pp. 1759-1768, 2016.

9. Dunne MP, Kelly S. OrthoFiller: utilising data from multiple species to improve the completeness of genome annotations. BMC Genomics. 2017;18(390)

10. Nasiri J, Naghavi M, Rad SN, Yolmeh T, Shirazi M, Naderi R, Nasiri M, Ahmadi S. Gene identification programs in bread wheat: a comparison study. Nucleosides Nucleotides Nucleic Acids. 2013;32(10):529-54.

11. Sudmant PH, Alexis MS, Burge CB. Meta-analysis of RNA-seq expression data across species, tissues and studies. Genome Biol. 2015;16(287)

12. Danielsson F, James T, Gomez-Cabrero D, Huss M. Assessing the consistency of public human tissue RNA-seq data sets. Brief Bioinform. 2015;16(6):941-9.

13. Conesa A, Madrigal P, Tarazona S, Gomez-cabrero D, Cervera A, Mcpherson A, Szcze W, Gaffney DJ, Elo LL, Zhang X. A survey of best practices for RNAseq data analysis. Genome Biol. 2016;17(13)

14. Wang L, Nie J, Sicotte H, Li Y, Eckel-passow JE, Dasari S, Vedell PT, Barman P, Wang L, Weinshiboum R, Jen J, Huang H, Kohli M, Kocher JA. Measure transcript integrity using RNA-seq data. BMC Bioinformatics. 2016;17(58)

15. Hansen KD, Brenner SE, Dudoit S. Biases in Illumina transcriptome sequencing caused by random hexamer priming. Nucleic Acids Res. 2010;38(12):e131.

16. Jiang $H$, Salzman J. A penalized likelihood approach for robust estimation of isoform expression. Stat Interface. 2015;8(4):437-45.

17. Abril JF, Castelo R, Guigó R. Comparison of splice sites in mammals and chicken. Genome Res. 2005;15(1):111-9.

18. Betts MJ, Guigó R, Agarwal P, Russell RB. Exon structure conservation despite low sequence similarity: a relic of dramatic events in evolution? EMBO J. 2001:20(19):5354-60.

19. Nurtdinov RN, Neverov AD, Favorov AV, Mironov AA, Gelfand MS. Conserved and species-specific alternative splicing in mammalian genomes. BMC Evol Biol. 2007;7:249. 
20. Alexandersson M, Cawley S, Pachter L. SLAM: cross-species gene finding and alignment with a generalized pair hidden Markov model. Genome Res. 2003;13:496-502.

21. Wiehe T, Gebauer-Jung S. SGP-1: prediction and validation of homologous genes based on sequence alignments. Genome Res. 2001;11:1574-83.

22. Gross SS, Brent MR. Using multiple alignments to improve gene prediction. J Comput Biol. 2006;13(2):379-93.

23. Gross SS, Do CB, Sirota M, Batzoglou S. CONTRAST: a discriminative, phylogeny-free approach to multiple informant de novo gene prediction. Genome Biol. 2007;8:R269.

24. Stanke M, Morgenstern B. "AUGUSTUS: a web server for gene prediction in eukaryotes that allows user-defined constraints," Nucleic Acids Res., vol. 33, no suppl_2. 2005:465-7.

25. Slater G, Birney E. Automated generation of heuristics for biological sequence comparison. BMC Bioinformatics. 2005;6(31)

26. Emms DM, Kelly S. OrthoFinder: solving fundamental biases in whole genome comparisons dramatically improves orthogroup inference accuracy. Genome Biol. 2015;16(157)

27. Goodstein DM, Shu S, Howson R, Neupane R, Hayes RD, Fazo J, Mitros T, Dirks W, Hellsten U, Putnam N, Rokhsar DS. Phytozome: a comparative platform for green plant genomics. Nucleic Acids Res. 2012;40:D1178-86.

28. R. Leinonen, H. Sugawara, and M. Shumway, "The sequence read archive," Nucleic Acids Res., vol. 39, no suppl_1, pp. D19-D21, 2010.

29. Katoh K, Standley DM. MAFFT multiple sequence alignment software version 7: improvements in performance and usability Article Fast Track. Mol Biol Evol. 2013;30(4):772-80.

30. Hagberg AA, Schult DA, Swart PJ. Exploring network structure, dynamics, and function using NetworkX. Proc 7th Python Sci Conf. 2008:11-6.

31. Pelechano $V$, Steinmetz LM. Gene regulation by antisense transcription. Nat Rev Genet. 2013;14:880-93.

32. S. Andrews, "FastQC: a quality control tool for high throughput sequence data," 2010. [Online]. Available: http://www.bioinformatics.babraham.ac.uk/ projects/fastqc.

33. H. Li, "Seqtk: Toolkit for processing sequences in FASTA/Q formats," 2012. [Online]. Available: https://github.com/lh3/seqtk. [Accessed: 17-Jan-2018].

34. D. Kim, B. Langmead, and S. Salzberg, "HISAT2: graph-based alignment of next-generation sequencing reads to a population of genomes." 2017.

35. Kim D, Langmead B, Salzberg SL. HISAT: a fast spliced aligner with low memory requirements. Nat Methods. 2015;12(4):357-62.

36. Li H, Handsaker B, Wysoker A, Fennell T, Ruan J, Homer N, Marth G, Abecasis $G$, Durbin R. The sequence alignment/map format and SAMtools. Bioinformatics. 2009:25(16):2078-9.

37. Emanuelsson $\mathrm{O}$, Nielsen $\mathrm{H}$, Brunak $\mathrm{S}$, von Heijne G. Predicting subcellular localization of proteins based on their $\mathrm{N}$-terminal amino acid sequence. J Mol Biol. Jul. 2000;300(4):1005-16.

38. Schneider $G$, Fechner $U$. Advances in the prediction of protein targeting signals. Proteomics. Jun. 2004;4(6):1571-80.

39. Goodstein DM, Shu S, Howson R, Neupane R, Hayes RD, Fazo J, Mitros T, Dirks W, Hellsten U, Putnam N, Rokhsar DS. Phytozome: a comparative platform for green plant genomics. Nucleic Acids Res. 2012;40(D1):1178-86.

40. Lamesch P, Berardini TZ, Li D, Swarbreck D, Wilks C, Sasidharan R, Muller R, Dreher K, Alexander DL, Garcia-Hernandez M, Karthikeyan AS, Lee CH, Nelson WD, Ploetz L, Singh S, Wensel A, Huala E. The Arabidopsis information resource (TAIR): improved gene annotation and new tools. Nucleic Acids Res. 2012;40:D1202-10.

41. R. Ming, S. Hou, Y. Feng, Q. Yu, A. Dionne-laporte, J. H. Saw, P. Senin, W. Wang, B. V Ly, K. L. T. Lewis, L. Salzberg, L. Feng, M. R. Jones, R. L. Skelton, J. E. Murray, W. Qian, J. Shen, P. Du, M. Eustice, E. Tong, E. Lyons, R. E. Paull, T. P. Michael, K. Wall, D. W. Rice, H. Albert, M. Wang, Y. J. Zhu, M. Schatz, N. Nagarajan, A. Acob, P. Guan, A. Blas, C. M. Wai, C. M. Ackerman, Y. Ren, C. Liu, J. Wang, J. Wang, J. Na, V. Eugene, B. Haas, J. Thimmapuram, D. Nelson, X. Wang, E. John, W. Zhang, G. Presting, A. Windsor, R. Navajas-pérez, J. Torres, F. A. Feltus, B. Porter, Y. Li, a M. Burroughs, L. Liu, D. a Christopher, S. M. Mount, and P. H. Moore, "The draft genome of the transgenic tropical fruit tree papaya (Carica papaya Linnaeus)," Nature, vol. 452, no. 7190, pp. 991-996, 2008.

42. Slotte T, Hazzouri KM, Ågren JA, Koenig D, Maumus F, Guo Y, Steige K, Platts AE, Escobar JS, Newman LK, Wang W, Mandáková T, Vello E, Smith LM, Henz SR, Steffen J, Takuno S, Brandvain Y, Coop G, Andolfatto P, Hu TT, Blanchette M, Clark RM, Quesneville H, Nordborg M. The Capsella rubella genome and the genomic consequences of rapid mating system evolution. Nat Genet. 2013;45(7):831-5.
43. Motamayor JC, Mockaitis K, Schmutz J, Haiminen N, III DL, Cornejo O, Findley SD, Zheng P, Utro F, Royaert S, Saski C, Jenkins J, Podicheti R, Zhao M, Scheffler BE, Stack JC, Feltus FA, Mustiga GM, Amores F, Phillips W, Marelli JP, May GD, Shapiro H, Ma J, Bustamante CD, Schnell RJ, Main D, Gilbert D, Parida L, Kuhn DN. The genome sequence of the most widely cultivated cacao type and its use to identify candidate genes regulating pod color. Genome Biol. 2013;14:r53.

44. Lindblad-Toh K, Wade CM, Mikkelsen TS, Karlsson EK, Jaffe DB, Kamal M, Clamp M, Chang JL, Kulbokas EJ, Zody MC, Mauceli E, Xie X, Breen M, Wayne RK, Ostrander EA, Ponting CP, Galibert F, Smith DR, DeJong PJ, Kirkness E, Alvarez P, Biagi T, Brockman W, Butler J, Chin CW, Cook A, Cuff J, Daly MJ, DeCaprio D, Gnerre S, Grabherr M, Kellis M, Kleber M, Bardeleben C, Goodstadt L, Heger A, Hitte C, Kim L, Koepfli KP, Parker HG, Pollinger JP, Searle SMJ, Sutter NB, Thomas R, Webber C, Lander ES. Genome sequence, comparative analysis and haplotype structure of the domestic dog. Nature. 2005;438(7069):803-19.

45. Benson DA, Cavanaugh M, Clark K, Karsch-Mizrachi I, Lipman DJ, Ostell J, Sayers EW. GenBank. Nucleic Acids Res. 2013;41:D36-42.

46. Pruitt KD, Harrow J, Harte RA, Wallin C, Diekhans M, Maglott DR, Searle S, Farrell CM, Loveland JE, Ruef BJ, Hart E, Suner MM, Landrum MJ, Aken B, Ayling S, Baertsch R, Fernandez-Banet J, Cherry JL, Curwen V, DiCuccio M, Kellis M, Lee J, Lin MF, Schuster M, Shkeda A, Amid C, Brown G, Dukhanina O, Frankish A, Hart J, Maidak BL, Mudge J, Murphy MR, Murphy T, Rajan J, Rajput B, Riddick LD, Snow C, Steward C, Webb D, Weber JA, Wilming L, Wu W, Birney E, Haussler D, Hubbard T, Ostell J, Durbin R, Lipman D. The consensus coding sequence (CCDS) project: identifying a common protein-coding gene set for the human and mouse genomes. Genome Res. 2009;19:1316-23.

47. Mikkelsen TS, Wakefield MJ, Aken B, Amemiya CT, Chang JL, Duke S, Garber M, Gentles AJ, Goodstadt L, Heger A, Jurka J, Kamal M, Mauceli E, Searle SMJ, Sharpe T, Baker ML, Batzer MA, Benos PV, Belov K, Clamp M, Cook A, Cuff J, Das R, Davidow L, Deakin JE, Fazzari MJ, Glass JL, Grabherr M, Greally JM, Gu W, Hore TA, Huttley GA, Kleber M, Jirtle RL, Koina E, Lee JT, Mahony S, Marra MA, Miller RD, Nicholls RD, Oda M, Papenfuss AT, Parra ZE, Pollock DD, Ray DA, Schein JE, Speed TP, Thompson K, VandeBerg JL, Wade CM, Walker JA, Waters PD, Webber C, Weidman JR, Xie X, Zody MC, Baldwin J, Abdouelleil A, Abdulkadir J, Abebe A, Abera B, Abreu J, Acer SC, Aftuck L, Alexander A, An P, Anderson E, Anderson S, Arachi H, Azer M, Bachantsang P, Barry A, Bayul T, Berlin A, Bessette D, Bloom T, Blye J, Boguslavskiy L, Bonnet C, Boukhgalter B, Bourzgui I, Brown A, Cahill P, Channer S, Cheshatsang Y, Chuda L, Citroen M, Collymore A, Cooke P, Costello M, D'Aco K, Daza R, De Haan G, DeGray S, DeMaso C, Dhargay N, Dooley K, Dooley E, Doricent M, Dorje P, Dorjee K, Dupes A, Elong R, Falk J, Farina A, Faro S, Ferguson D, Fisher S, Foley CD, Franke A, Friedrich D, Gadbois L, Gearin G, Gearin CR, Giannoukos G, Goode T, Graham J, Grandbois E, Grewal S, Gyaltsen K, Hafez N, Hagos B, Hall J, Henson C, Hollinger A, Honan T, Huard MD, Hughes L, Hurhula B, Husby ME, Kamat A, Kanga B, Kashin S, Khazanovich D, Kisner P, Lance K, Lara M, Lee W, Lennon N, Letendre F, LeVine R, Lipovsky A, Liu X, Liu J, Liu S, Lokyitsang T, Lokyitsang $Y$, Lubonja R, Lui A, MacDonald P, Magnisalis V, Maru K, Matthews C, McCusker W, McDonough S, Mehta T, Meldrim J, Meneus L, Mihai O, Mihalev A, Mihova T, Mittelman R, Mlenga V, Montmayeur A, Mulrain L, Navidi A, Naylor J, Negash T, Nguyen T, Nguyen N, Nicol R, Norbu C, Norbu N, Novod N, O'Neill B, Osman S, Markiewicz E, Oyono OL, Patti C, Phunkhang P, Pierre F, Priest M, Raghuraman S, Rege F, Reyes R, Rise C, Rogov P, Ross K, Ryan E, Settipalli S, Shea T, Sherpa N, Shi L, Shih D, Sparrow T, Spaulding J, Stalker J, StangeThomann N, Stavropoulos S, Stone C, Strader C, Tesfaye S, Thomson T, Thoulutsang Y, Thoulutsang D, Topham K, Topping I, Tsamla T, Vassiliev $H$, Vo A, Wangchuk T, Wangdi T, Weiand M, Wilkinson J, Wilson A, Yadav S, Young G, Yu Q, Zembek L, Zhong D, Zimmer A, Zwirko Z, Jaffe DB, Alvarez P, Brockman W, Butler J, Chin CW, Gnerre S, MacCallum I, Graves JAM, Ponting CP, Breen M, Samollow PB, Lander ES, Lindblad-Toh K. Genome of the marsupial Monodelphis domestica reveals innovation in non-coding sequences. Nature. 2007;447(7141):167-77.

48. Alföldi J, Di Palma F, Lindblad-toh K. The European rabbit genome. In: Rabbit biotechnology: rabbit genomics, Transgenesis, cloning and models; 2009. p. 129.

49. F. S. Fred S. Dietrich, S. Voegeli, S. Brachat, A. Lerch, K. Gates, S. Steiner, C. Mohr, P. Luedi, S. Choi, R. A. Wing, A. Flavier, T. D. Gaffney, P. Philippsen, P. Fred S. 
Dietrich, Fred S Voegeli S Brachat S Lerch A Gates K Steiner S. and Mohr C Luedi P Choi S wing r a Flavier A and Gaffney T D and Philippsen, "the Ashbya gossypii genome as a tool for mapping the ancient Saccharomyces cerevisiae genome," Science (80-.) )., vol. 304, no. April, 2004.

50. Dujon B, Sherman D, Fischer G, Durrens P, Casaregola S, Lafontaine I, De Montigny J, Blanchin S, Beckerich J-M, Beyne E, Bleykasten C, Babour A,

Boyer J, Cattolico L, Confanioleri F, De Daruvar A, Despons L, Fabre E, De Montigny J, Marck C, Neuvéglise C, Talla E, Goffard N, Frangeul L, Aigle M, Anthouard V, Babour A, Barbe V, Barnay S, Blanchin S, Beckerich J-M, Beyne E, Bleykasten C, Boisramé A, Boyer J, Cattolico L, Confanioleri F, De Daruvar A, Despons L, Fabre E, Fairhead C, Ferry-Dumazet H, Groppi A, Hantraye F, Hennequin C, Jauniaux N, Joyet P, Kachouri R, Kerrest A, Koszul R, Lemaire M, Lesur I, Ma L, Muller H, Nicaud J-M, Nikolski M, Oztas S, OzierKalogeropoulos O, Pellenz S, Potier S, Richard G-F, Straub M-L, Suleau A, Swennen D, Tekaia F, Wésolowski-Louvel M, Westhof E, Wirth B, ZeniouMeyer M, Zivanovic I, Bolotin-Fukuhara M, Thierry A, Bouchier C, Caudron B, Scarpelli C, Gaillardin C, Weissenbach J, Wincker P, Souciet J-L. Genome evolution in yeasts. Nature. 2004;430(6995):35-44.

51. Sacerdot C, Casaregola S, Lafontaine I, Tekaia F, Dujon B, Ozierkalogeropoulos $\mathrm{O}$. Promiscuous DNA in the nuclear genomes of hemiascomycetous yeasts. FEMS Yeast Res. 2008;8:846-57.

52. Gnerre S, Maccallum I, Przybylski D, Ribeiro FJ, Burton JN, Walker BJ, Williams L, Nicol R, Gnirke A, Nusbaum C, Lander ES, Jaffe DB, Sharpe T, Hall G, Shea TP, Sykes S, Berlin AM, Aird D, Costello M, Daza R, Williams L, Nicol R, Gnirke A, Nusbaum C, Lander ES, Jaffe DB. High-quality draft assemblies of mammalian genomes from massively parallel sequence data. PNAS. 2011;108(4):1513-8.

Ready to submit your research? Choose BMC and benefit from:

- fast, convenient online submission

- thorough peer review by experienced researchers in your field

- rapid publication on acceptance

- support for research data, including large and complex data types

- gold Open Access which fosters wider collaboration and increased citations

- maximum visibility for your research: over $100 \mathrm{M}$ website views per year

At BMC, research is always in progress.

Learn more biomedcentral.com/submissions 